\title{
Resequencing the susceptibility gene, ITGAM, identifies two functionally deleterious rare variants in systemic lupus erythematosus cases
}

Amy L Roberts ${ }^{1}$, Ellen RA Thomas², Shriram Bhosle², Laurence Game², Olga Obraztsova', Timothy J Aitman², Timothy J Vyse ${ }^{1 *}$ and Benjamin Rhodes ${ }^{1}$

\begin{abstract}
Introduction: The majority of the genetic variance of systemic lupus erythematosus (SLE) remains unexplained by the common disease-common variant hypothesis. Rare variants, which are not detectable by genome-wide association studies because of their low frequencies, are predicted to explain part of this "missing heritability." However, recent studies identifying rare variants within known disease-susceptibility loci have failed to show genetic associations because of their extremely low frequencies, leading to the questioning of the contribution of rare variants to disease susceptibility. A common (minor allele frequency $=17.4 \%$ in cases) nonsynonymous coding variant rs 1143679 (R77H) in ITGAM (CD11b), which forms half of the heterodimeric integrin receptor, complement receptor 3 (CR3), is robustly associated with SLE and has been shown to impair CR3-mediated phagocytosis.

Methods: We resequenced ITGAM in 73 SLE cases and identified two previously unidentified, case-specific nonsynonymous variants, F941V and G1145S. Both variants were genotyped in 2,107 and 949 additional SLE cases, respectively, to estimate their frequencies in a disease population. An in vitro model was used to assess the impact of F941V and G1145S, together with two nonsynonymous ITGAM polymorphisms, A858V (rs1143683) and M441T (rs1 1861251), on CR3-mediated phagocytosis. A paired two-tailed $t$ test was used to compare the phagocytic capabilities of each variant with that of wild-type CR3.
\end{abstract}

Results: Both rare variants, F941V and G1145S, significantly impair CR3-mediated phagocytosis in an in vitro model (61\% reduction, $P=0.006 ; 26 \%$ reduction, $P=0.0232$ ). However, neither of the common variants, M441T and A858V, had an effect on phagocytosis. Neither rare variant was observed again in the genotyping of additional SLE cases, suggesting that their frequencies are extremely low.

Conclusions: Our results add further evidence to the functional importance of ITGAM in SLE pathogenesis through impaired phagocytosis. Additionally, this study provides a new example of the identification of rare variants in common-allele-associated loci, which, because of their extremely low frequencies, are not statistically associated. However, the demonstration of their functional effects adds support to their contribution to disease risk, and questions the current notion of dismissing the contribution of very rare variants on purely statistical analyses.

\footnotetext{
* Correspondence: Timothy.vyse@kcl.ac.uk

${ }^{1}$ Department of Medical and Molecular Genetics, and Inflammation, Infection and Immunity, King's College London, London SE1 9RT, UK

Full list of author information is available at the end of the article
} 


\section{Introduction}

Systemic lupus erythematosus (SLE) is a phenotypically heterogeneous autoimmune disease resulting from the breakdown of immune tolerance. SLE has a strong and complex genetic component, as shown by estimates of twin concordance [1]. Although 52 genetic loci now are independently associated with SLE, the vast majority of which are products of the genome-wide technologic advancements of recent years, only an estimated $16 \%$ of the total variance is thought to be explained [2,3] (J. Bentham, D. L. Morris, et al. 2014, unpublished data].

The genome-wide association study (GWAS) era has uncovered a plethora of common alleles, mostly of modest effect sizes (odds ratio $(\mathrm{OR}),<2$ ), associated with complex diseases under the common disease-common variant (CDCV) hypothesis [4]. Yet for most of these diseases, as with SLE, the amount of remaining unexplained genetic variance (the so-called missing heritability) is higher than initially anticipated [5-7]. Rare variants with larger effect sizes $(\mathrm{OR}, \geq 2)$, but present at frequencies below the detectable threshold of GWAS $(<5 \%)$, have been hypothesized to explain part of this "missing" genetic effect $[8,9]$. Although they are individually rare, they are collectively common and therefore may make a significant contribution to disease risk on a population level.

Because of their low frequency and incomplete penetrance, individual rare variants may never reach statistically significant levels of association, irrespective of very large sample sizes [10]. Furthermore, the relatively high de novo mutation rate in humans [11], means that chromosomes of unaffected individuals will also harbor rare, or even private, mutations that may or may not influence disease risk. Genome- or exome-wide sequencing may uncover many new variants, but determining which of these are functionally relevant is not easy by genetic analysis alone [12]. One approach is to use the notable common variant discoveries of GWAS to focus on resequencing candidate genes that are already known to contain common disease-risk variants. Many studies have now identified additional rare variants in GWAS-associated loci $[13,14]$. The largest of such studies focused on the coding exons of 25 autoimmune GWAS-associated loci and concluded that a negligible affect from rare variants was present [15]. Ultimately, however, functional analyses of rare variants will best enable us to understand the importance of associated loci in disease pathogenesis and add confirmatory evidence to disease biology paradigms established by the functional effect of common variants.

SLE is robustly associated with ITGAM (ENSG00000 169896), which encodes CD11b [16]. Through noncovalent binding with $\mathrm{CD} 18$, this forms the heterodimeric integrin complement receptor 3 (CR3; Mac-1; CD11b/CD18), expressed on phagocytes and NK cells [17]. Despite the high degree of linkage disequilibrium
(LD) across the ITGAM locus in European populations, transancestral mapping provided evidence that the common nonsynonymous polymorphism rs1143679, encoding an arginine-to-histidine amino acid change at codon 77 of CD11b (R77H), was the true causal SNP [18]. Subsequently, functional evidence supported this genetic association, showing that $\mathrm{R} 77 \mathrm{H}$ impairs many functions of the CR3 receptor, including the phagocytosis of iC3b-coated targets by ex vivo monocyte-derived macrophages [19], neutrophils [20], and in vitro models [21]. In addition to rs1143679, speculation has occurred about a secondary independent SLE association within ITGAM due to a second nonsynonymous variant rs1143683 (A858V) [22]. Because precedence exists for the presence of multiple effects in risk loci of complex diseases, this requires further evaluation [23].

Here we report the identification of two novel, casespecific rare variants in ITGAM after resequencing of this locus in cases of European ancestry. Both variants, F941V and G1145S, were functionally damaging, as measured by an in vitro model of CR3-mediated phagocytosis, but the common A858V (rs1143683) variant was functionally neutral. Furthermore, neither F941V nor G1145S was observed in the follow-up genotyping of 2107 and 949 SLE cases, respectively. This study shows the importance of multiple approaches to the analysis of rare variants, and the need for better methods to estimate contributions, before we dismiss the importance of this large body of genetic variation.

\section{Methods}

\section{The 454 sequencing and analysis}

Genomic DNA from 73 SLE patients meeting American College of Rheumatology criteria underwent whole-genome amplification (Qiagen Repli-G, Hilden, Germany). Ethical approval was given by London Multicentre Research Ethics Committee, and participants gave written consent. Standard (KOD polymerase, Merck) and long-range (Sequalprep, Invitrogen, CA, USA) PCR reactions were carried out in 13 amplicons, giving products between 600 base pairs and 4 $\mathrm{kb}$ in length, covering $24 \mathrm{~kb}$ of the ITGAM gene, including all 30 exons (see Additional file 1). The PCR products were run on an agarose gel and purified by using the Qiagen MinElute 96 UF PCR Purification Kit. Seven amplicons greater than $1.5 \mathrm{~kb}$ were pooled and sheared to fragments of 500 to $800 \mathrm{bp}$ on the Covaris E210. The six short PCR products were added to the sheared products, and each pool was tagged with a unique DNA barcode by using the Parallel Tagged Sequencing (PTS) protocol [24], starting at the blunt-end repair step. The barcoded PCR products were quantified by using the Invitrogen Quant-iT Picogreen dsDNA kit and combined into a single pool by using $10 \mathrm{ng}$ of each tagged sample.

Dephosphorylation and restriction digestion was carried out according to the PTS protocol, and the pool of 
sheared and indexed PCR products was taken through 454 Titanium library preparation according to the manufacturer's protocol (GS FLX Titanium General Library Preparation Method Manual, v5.3.2), starting at step 3.5. Library quality was checked by using an RNA Pico 6000 assay on the Agilent Bioanalyser and quantified by using a RiboGreen assay (Thermo Fisher Scientific, MA, USA) with the Nanodrop 3300 fluorospectrometer. The library was diluted to $1 \times 10^{8}$ molecules/ $\mu \mathrm{l}$, amplified and sequenced according to the manufacturer's protocol for 454 GS FLX sequencing. With 896,206 reads that passed filters, an average coverage of $105 \times$ was achieved; $88 \%$ of the samples were covered at a level sufficient for variant calling (more than 10 reads) in at least $80 \%$ of the target region.

Additional PCRs (KOD polymerase, Merck, Darmstadt, Germany) were generated covering exons 8, 13 and 14 due to low coverage in the 454 data. Capillary sequencing of these amplicons used the 3730xl platform (Applied Biosystems, CA, USA).

The 454 sequence read demultiplexing used "untag" software [24]; base calling used Pyrobayes [25]; and alignment to the reference genome used BWA [26]. Variant calling was carried out by using GATK [27] without duplicate read removal, by using hard filters: coverage at least 10fold; genotype quality threshold, 50; SNP quality threshold, 30; and allele balance threshold, 0.75 .

The presence of novel variants was confirmed independently by PCR amplification of native genomic DNA followed by capillary sequencing (see Additional file 2). G1145S was genotyped in 949 additional SLE cases with capillary sequencing by using the following primers: 5 ' GGCTTCTTCAAGCGGCAATA and 5' GTCCTGTCG GGGATACTTCG. F941V was genotyped in 2,107 additional SLE cases on an Illumina Custom 384 chip.

\section{Site-directed mutagenesis}

10 ng of a pcDNA3.1-CD11b vector (Life technologies, Paisley, UK) was mutated to contain one of four ITGAM variants by using the PCR-based QuikChange II XL Site-Directed Mutagenesis kit (Agilent Technologies, Stockport, UK), followed by transformation of XL-10 Gold Ultra-competent cells, as per the manufacturer's instructions. The primers used for the introduction of each of the variants were as follows: M441T $5^{\prime}$-gcagaacactgg cacgtgggagtccaacg-3' and 5' -cgttggactcccacgtgccagtgttctgc3'; A858V 5' -ccgaagtgtctggggtcttgaagagcaccag-3' and 5'ctggtgctcttcaagacccagacacttcgg-3'; F941V 5' -tctccactaaa tatctcaacgtcacggcctcagagaat- $3^{\prime}$ and $5^{\prime}$-attctctgaggccgtgacg ttgagatatttagtggaga-3'; G1145S 5' -atgagtgaagggagtccccog ggg-3' and $5^{\prime}$-ccccgggggactcccttcactcat-3'.

\section{Transient transfections}

COS-7 cells (ATCC, USA) were maintained in 10\% FBS DMEM (Life Technologies); $1 \times 10^{6}$ cells were co- transfected with one of five pcDNA3.1-CD11b vectors (WT, M441T, A858V, F841V, or G1145S) together with $1.25 \mathrm{~g}$ pcDNA3.1-CD18 by using nucleofection (Nucleofector II; Lonza, Basel, Switzerland). In duplicate, $40 \times 10^{4}$ cells were added to an acid-cleaned coverslip in a 24-well plate. The remaining transfected cells were cultured for expression screening with flow cytometry.

\section{Flow cytometry}

One or two days after transfection, cell-surface expression of CR3 was measured by using a PE-conjugated antiCD11b (clone ICRF44) antibody and PE-conjugated isotype control. Transfected cells were stained in the dark for 30 minutes on ice and processed on a BD FACS Canto II flow cytometer. The percentage of CR3-positive cells was estimated, and the mean fluorescence intensity (MFI) measured, by using FlowJo software v.7.6.4. A paired $t$ test was used to compare the MFI of the CR3-positive cell population. No significant difference in cell-surface expression of CD11b was observed between WT $(n=5$, mean \pm SD; 14,445 $\pm 6,982)$ and M441T $(15,017 \pm 7,993)$, A858V (12,377 $\pm 5,130)$, F941V (14,327 $\pm 4,358)$, or G1145S $(12,767 \pm 5,028)$ CD11b transfected cells.

\section{Phagocytic assay}

This was carried out on the same day as the flow-cytometry expression analysis. The method used is identical to that in our previously published work [19] and has been used extensively as an in vitro model for phagocytic receptors $[28,29]$. In summary, sheep red blood cells (sRBCs) were coated with anti-sheep IgG at room temperature for 1 hour, followed by incubation at $37^{\circ} \mathrm{C}$ for 20 minutes with C5-depleted serum to form iC3b-opsinized sRBC $\left(\mathrm{sRBC}_{\mathrm{iC} 3 \mathrm{~b}}\right)$. Transfected COS-7 cells were challenged with $\mathrm{sRBC}_{\mathrm{iC} 3 \mathrm{~b}}$ for 30 minutes at $37^{\circ} \mathrm{C}$. External sRBC $\mathrm{C}_{\mathrm{iC} 3 \mathrm{~b}}$ were labeled with an Alexa-488 conjugated anti-IgG antibody and, after fixation and permeabiliation, both internal and external $\mathrm{sRBC}_{\mathrm{iC} 3 \mathrm{~b}}$ were labeled with an Alexa555conjugated anti-IgG antibody. COS-7 nuclei were stained with DAPI. A Zeiss Axiophot fluorescent microscope was used visually to analyze the coverslips.

\section{Analysis}

Associated RBCs are double-stained with Alexa-488 and Alexa-555 and are therefore visible under both fluorescent filters. A phagocytic event was characterized by the absence of the Alexa-488 stain. We measured the association index (AI, mean number of engaged $\mathrm{sRBC}_{\mathrm{iC} 3 \mathrm{~b}} / 100 \mathrm{COS}$ cells), phagocytic index (PI, mean number of phagocytosed $\mathrm{sRBC}_{\mathrm{iC} 3 \mathrm{~b}} / 100 \mathrm{COS}$ cells), and percentage phagocytosis (\% $\mathrm{P}$, mean percentage phagocytosis/COS cell). A mean number of 67 COS-7 cells were counted from at least two coverslips per assay. Comparisons were made between WT 
and variants by using paired two-tailed $t$ tests on Prism 5 software (GraphPad, CA, USA).

\section{Results}

Two novel non-synonymous variants discovered through ITGAM resequencing

We used a candidate-gene approach and resequenced ITGAM by using the high-throughput 454 GS FLX platform in 73 SLE cases in search of putative rare variants. Then $24 \mathrm{~Kb}$ of the ITGAM locus was sequenced, covering all 30 exons and every intron/exon boundary. Two rare novel variants were identified in one patient sample each, F941V and G1145S (Table 1), and were confirmed by capillary sequencing after PCR amplification of native genomic DNA (Additional file 2). F941V was further genotyped on an Illumina Custom 384 Chip in 2,107 SLE cases of European ancestry. G1145S did not pass QC analysis of this genotyping method, possibly because of its close proximity ( $3 \mathrm{bp}$ ) to the common polymorphism P1146S, and so was genotyped with capillary sequencing in 949 SLE cases of European ancestry. Neither variant was observed again in these additional cases; therefore, we can estimate the minor allele frequencies of these variants to be $\mathrm{F} 941 \mathrm{~V}$, $\leq 0.0002$, and G1145S, $\leq 0.0005$.

CD11b codons 941 and 1145 are located in the membrane-proximal extracellular domain and the short cytoplasmic tail of the protein, respectively (Figure 1). Table 1 shows the in silico estimates of pathogenicity by using PolyPhen and SIFT. F941V was predicted to be deleterious, and G1145S was predicted to be benign, by both algorithms.

We used two large-scale publicly available sequencing project datasets to screen ITGAM on the chromosomes of healthy controls of European ancestry. Neither variant, F941V or G1145S, is described in the 1,000 chromosomes of European ancestry in the 1,000 Genomes project [32], nor are they described in the 8,600 European-American chromosomes of The National Heart Lung and Blood Institute (NHLBI) Exome Variant Server [33]. Therefore, both variants are novel, and, if not private, they are present at a frequency below 0.0001 in healthy individuals of European ancestry. It is worth noting that neither variant is found in the other populations included in the databases. This was expected, given that rare variants will be population specific.

MultAlin [34,35] was used to compare the protein sequences of the four CD11 molecules that pair with CD18 as part of the $\beta_{2}$ integrin family (Figure 2 ). The four proteins, CD11a, CD11b, CD11c, and CD11d, are encoded by ITGAX, ITGAM, ITGAL, and ITGAD, respectively. The phenylalanine $(\mathrm{F})$ residue at $\mathrm{CD} 11 \mathrm{~b}$ codon 941 , which is located in the membrane-proximal extracellular domain, is conserved across all four polypeptide sequences. The Glycine (G) residue at CD11b codon 1,145 is not shared with any of the other three CD11 proteins. No homology occurs at this relative position among any of the CD11 amino acid sequences. The short cytoplasmic tails of the CD11 molecules vary in length and sequence, and the differences observed are likely to be crucial for their varying functions, and/or intracellular signaling properties. Data from the UCSC Genome Browser [36] indicated that both CD11b residues, F941 and G1145, are highly conserved across mammalian species (data not shown).

\section{In vitro analyses of rare and common variants}

We used transiently transfected COS-7 cells to measure the effect of newly identified ITGAM rare variants on the binding and phagocytosis of iC3b-coated sheep red blood cells $\left(\mathrm{sRBC}_{\mathrm{iC} 3 \mathrm{~b}}\right)$, in addition to CR3 cell-surface expression. Additionally, we included the two common polymorphisms M441T (rs11861251) and A858V (rs1143683), which are in low $\left(r^{2}=0.017\right)$ and relatively high $\left(r^{2}=\right.$ 0.551) LD, respectively, with R77H in European populations, in our in vitro analyses. LD scores were calculated with 1000 Genomes Pilot 1 CEU data set by using SNAP Pairwise LD [37].

To test whether the nonsynonymous variants affected iC3b binding, we measured the ability of transiently transfected COS-7 cells to associate with iC3b-opsinized sheep red blood cells $\left(\mathrm{sRBC}_{\mathrm{iC} 3 \mathrm{~b}}\right)$ by using an Association Index. No significant difference was observed when variant CD11b was compared with WT, indicating that none of the four variants (M441T $P=0.79$; A858V $P=0.69$; F941V $P=0.61$; G1145S $P=0.85)$ affects the iC3b-binding ability of CR3 (Figure 3). Given that the four variants are located outside the $\mathrm{iC} 3 \mathrm{~b}$-binding site of the protein (Figure 1), this was an expected result.

Table 1 Summary of common polymorphisms and rare variants used for in vitro study

\begin{tabular}{lcccc}
\hline Variant & Chromosomes from resequencing study with minor allele (total = 146) & PolyPhen-2 & SIFT \\
\hline M441T & 21 & Benign (0) & Tolerated (0.63) \\
A858V & 33 & Benign (0.001) & Tolerated (0.35) \\
F941V & 1 & Probably damaging (1.00) & Damaging (0) \\
G1145S & 1 & Benign (0.319) & Tolerated (0.5) \\
\hline
\end{tabular}

PolyPhen-2 [30] categorizes mutations as benign, possibly damaging, or probably damaging, based on pairs of false-positive rate (FPR) thresholds. SIFT [31] predicts variants to be deleterious, given an output value $<0.05$. 


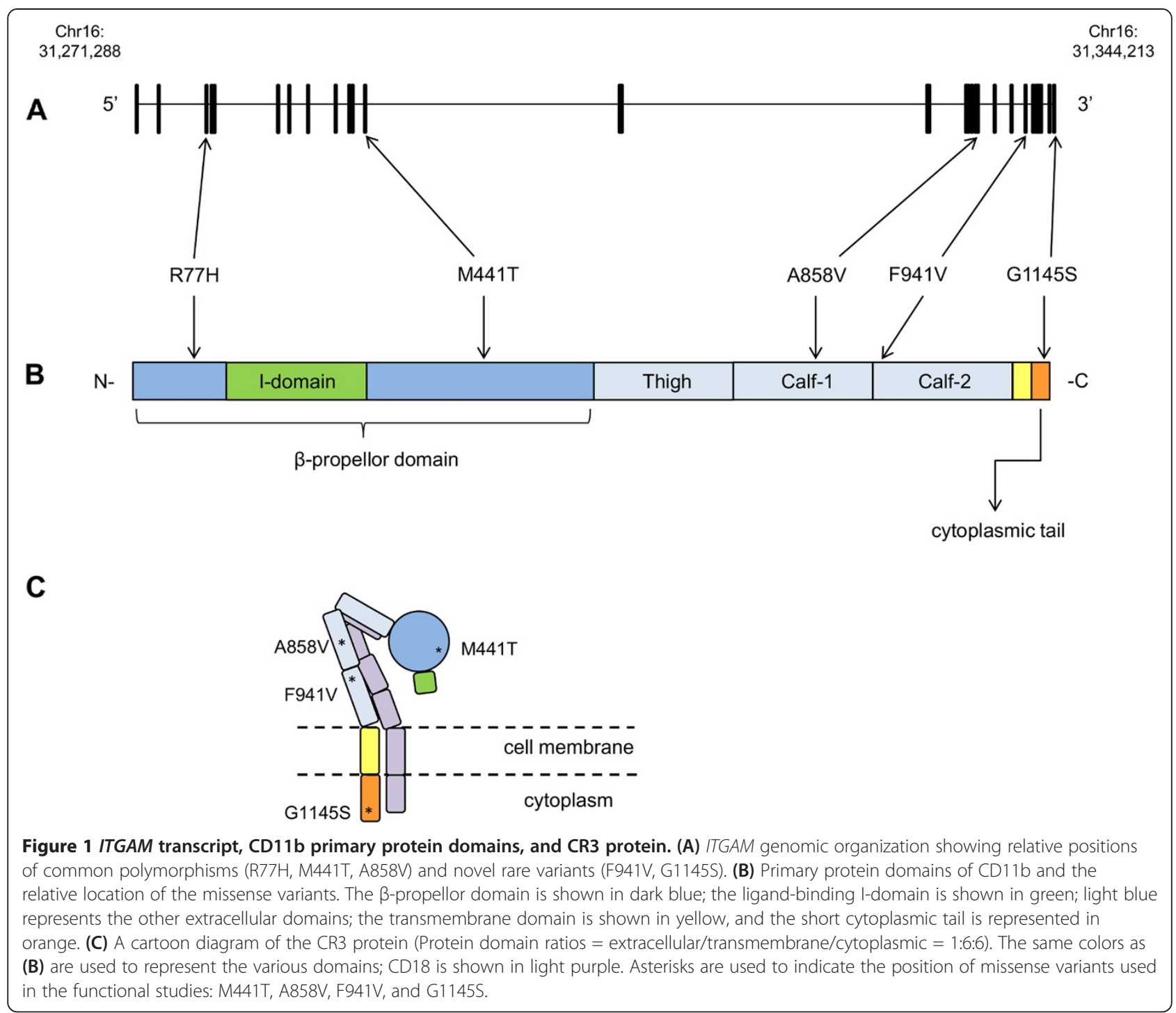

F941V significantly impaired the Phagocytic Index $(P=$ 0.002 ; Figure 4$)$ and the percentage phagocytosis $(P=$ 0.006 ; Figure 5), and the G1145S variant significantly impaired percentage phagocytosis $(P=0.0232$; Figure 5$)$, when compared with the WT (see Additional file 3). Neither of the two common polymorphisms had an effect on the phagocytosis of $\mathrm{sRBC} \mathrm{C}_{\mathrm{iC} 3 \mathrm{~b}}$ as measured by the Phagocytic Index (M441T, $P=0.74 ; \mathrm{A} 858 \mathrm{~V}, P=0.92$; Figure 4 ) and percentage phagocytosis (M441T, $P=0.70$; A858V, $P=0.47$; Figure 5).

The same transient transfection COS-7 model had previously been used by our group to demonstrate the deleterious effects of the SLE-associated missense polymorphism, R77H, on iC3b-dependent phagocytosis [19]. We used the mean reduction in percentage phagocytosis to compare the results of this study with that of the previous R77H study (Figure 6). As seen here, G1145S (26\%) has a comparable magnitude of effect to that of R77H (31\%), whereas F941V (61\%) has an even greater effect on phagocytosis.

\section{Discussion}

Through resequencing ITGAM in SLE patients of European ancestry, we identified two novel, case-specific variants. The in vitro model shows that both these variants, G1145S and, more strongly, F941V, impair CR3-mediated phagocytosis of iC3b-coated targets. The observed detrimental impact on protein function is of a similar (G1145S), or greater (F941V), magnitude to that of the common SLE-associated polymorphism R77H (which was associated with a $31 \%$ reduction in phagocytosis in an earlier article) [19]. Two factors support the importance of 


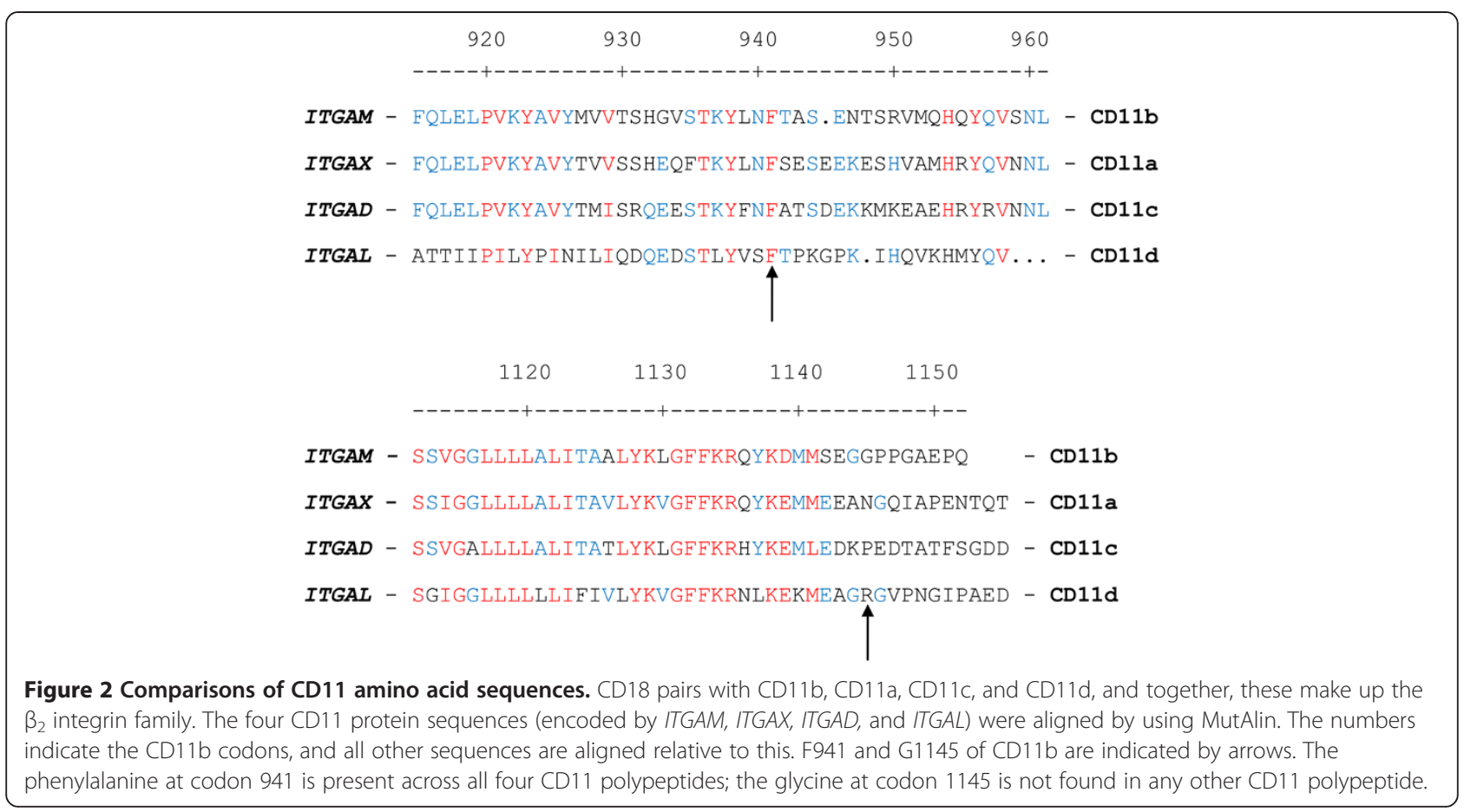

our data: first, they add further evidence for the coexistence of common and rare variants in genetic loci predisposing to the same disease biology, and second, they emphasize the role of underfunctioning ITGAM variants in SLE pathogenesis.
The targeted resequencing of loci harboring diseaseassociated common polymorphisms has successfully identified novel rare variants in a number of other complex genetic diseases such as inflammatory bowel disease, psoriasis, and asthma [13,14,38]. Recent studies in schizophrenia
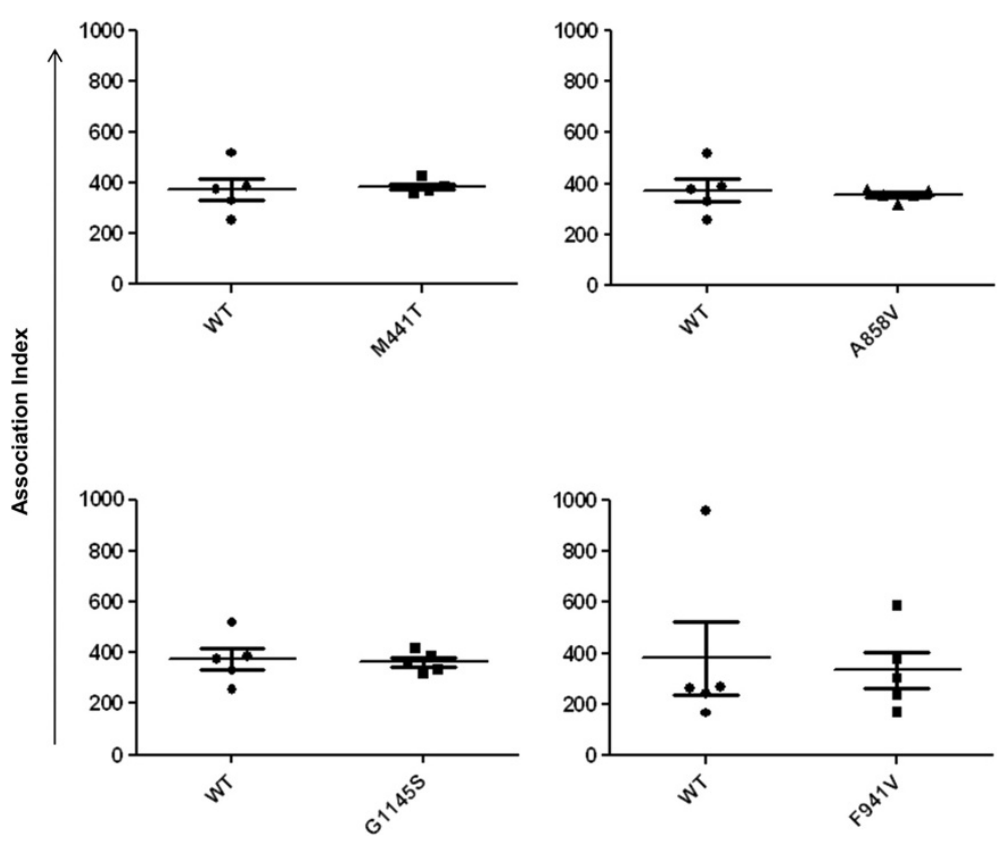

Figure 3 Association of CR3-expressing COS-7 cells with sRBC $\mathbf{~}_{\mathbf{i c} 3 \mathbf{b}}$. Association Index = mean number of associated (internal and external) $\mathrm{SRBC}_{\mathrm{ic} 3 \mathrm{~b}} / 100$ COS-7 cells. No significant difference was observed between WT and M441T $(P=0.79), \mathrm{A} 858 \mathrm{~V}(P=0.65), \mathrm{G} 1145 \mathrm{~S}(P=0.85)$, or F941V $(P=0.61)$. 


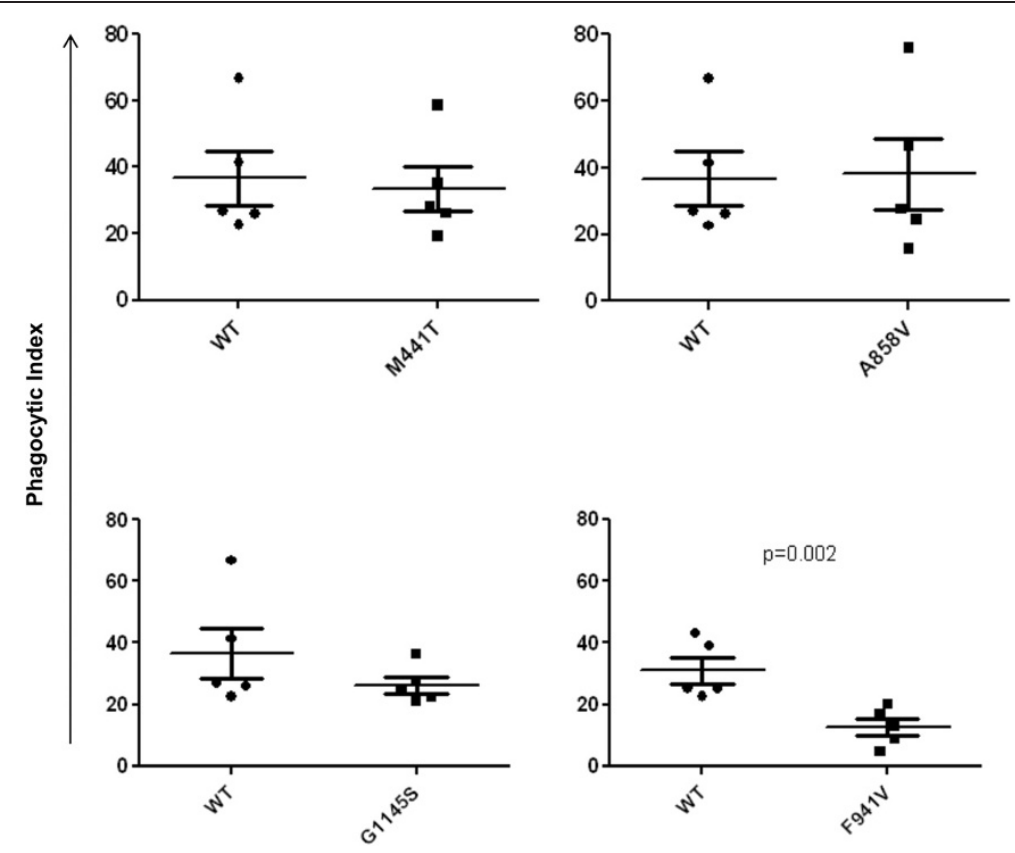

Figure 4 Phagocytosis of $\mathrm{sRBC}_{\mathrm{ic} 3 \mathrm{~b}}$ by CR3-expressing COS-7cells as measured with the Phagocytic Index. Phagocytic Index, mean number of internal sRBC $\mathrm{iC3b} / 100$ COS-7 cells. F941V significantly impairs phagocytosis $(P=0.002)$. No significant difference observed between WT and either common polymorphism M441T $(P=0.74)$ and A858V $(P=0.92)$, or the rare variant G1145S $(P=0.37)$.
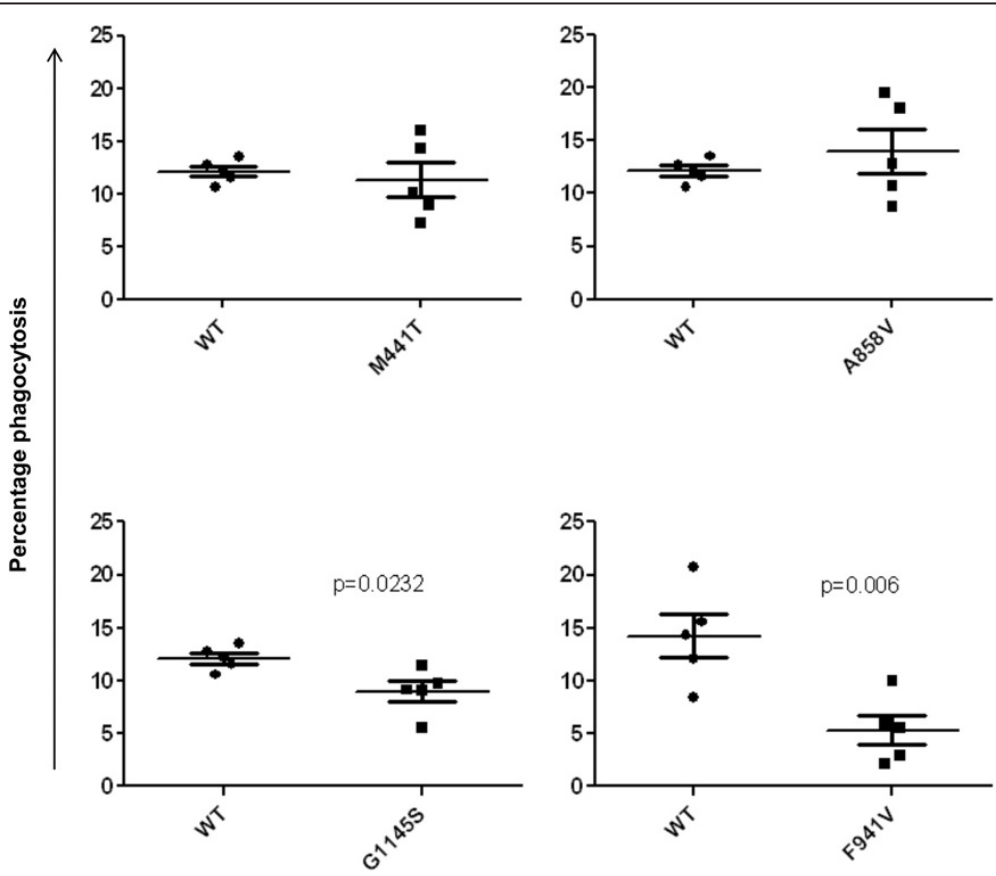

Figure 5 Phagocytosis of $\mathbf{s R B C} \mathrm{C}_{\mathrm{ic} 3 \mathrm{~b}}$ by CR3-expressing COS-7cells as measured with percentage phagocytosis. Percentage phagocytosis, mean percentage phagocytosis/COS-7 cell. Both rare variants F941V and G1145S significantly impair phagocytosis $(P=0.006$ and 0.0232 , respectively). No significant difference was observed between $\mathrm{WT}$ and either common polymorphism M441T $(P=0.70)$ and A858V $(P=0.47)$. 


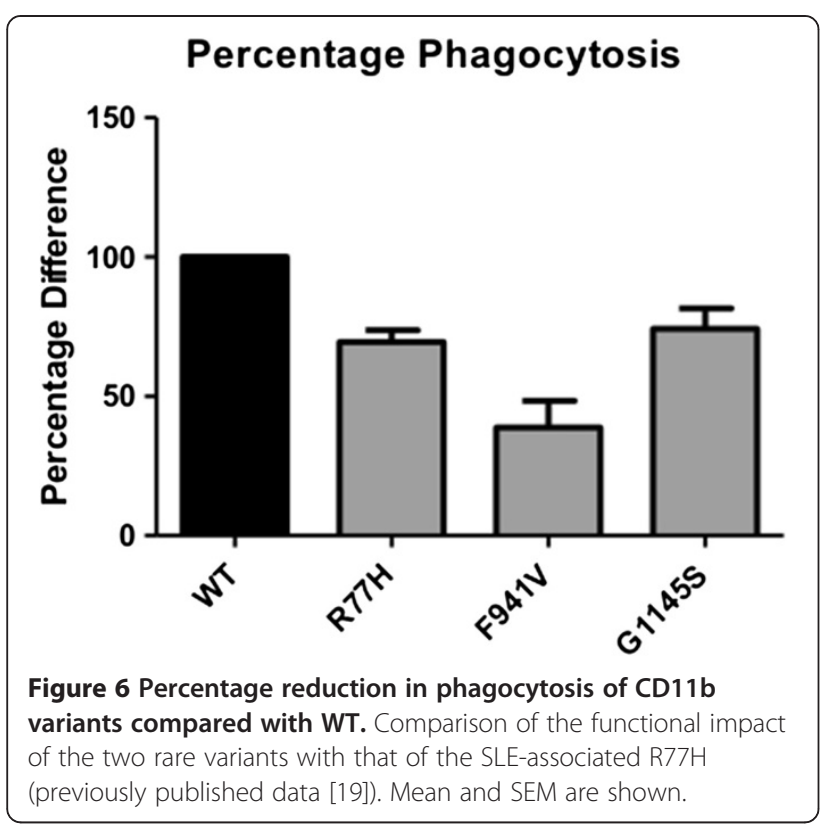

[39] and epilepsy [10] identified rare variants through whole-exome sequencing but failed to identify a single variant that reached study-wide significance, despite large-scale genotyping in follow-up cohorts. The schizophrenia study reported that $23 \%$ of case-specific variants from the initial sequencing cohort were not observed again in the followup cohort [39]. As demonstrated by these two studies, individual rare variants may never reach statistical significance for disease association. In our follow-up genotyping, 2,107 and 949 additional SLE cases were found to be wild-type for F941V and G1145S, respectively.

To assess the contribution of these novel ITGAM variants to disease biology, we additionally estimated their functional impact. We were able to do this by using a robust functional assay [28] that had previously proven successful in delineating function of the common ITGAM R77H variant [19]. Our work does not contribute to the estimate of overall genetic variance explained for SLE, but it does add evidence to the functional importance of the ITGAM locus to SLE susceptibility.

Furthermore, this study is another example of rare variants located in genetically associated loci whose contribution to disease risk cannot be estimated by statistical models alone because of their extremely low frequencies. However, given the demonstrated functional impact of these variants, their contribution cannot be dismissed. A case report of a CR3 defect in an SLE patient was previously assumed to be a result of a rare genetic variant [40]. Until additional methods of analysis are available, the contribution of rare variants to complex diseases should not be dismissed on the basis of non-significant statistical associations alone.
Although we observed clear functional similarities between the novel rare variants and $\mathrm{R} 77 \mathrm{H}$, the magnitude of this functional effect was different between variants, being stronger for variant F941V than for G1145S or common variant $\mathrm{R} 77 \mathrm{H}$. This raises an important point about the rare variant paradigm, which is often represented as a direct negative correlation between allele frequencies and disease risk [41]. Our in vitro model indicates that within a single gene, functional variants may vary considerably both in frequency ( $\mathrm{MAF}=0.2$ versus MAF $\leq 0.0005)$ and functional magnitude.

Defective phagocytosis is a robust paradigm in SLE biology [42], but of course may not be the only mechanism by which impaired CR3 function predisposes to SLE risk. It is possible, then, that F941V and/or G1145S have variable effect sizes on other CR3 functions, such as the inhibition of Toll 7/8-induced proinflammatory cytokines; however, in the absence of ex vivo cells from multiple healthy individuals carrying these mutations, or additional suitable in vitro assays, we lack a sufficient model for this $[19,43]$. We recognize that it is difficult to estimate directly the contribution of these rare variants to SLE risk; however, we believe that because their impact on phagocytosis is equal to, or greater than, that of $\mathrm{R} 77 \mathrm{H}$, their effect should not be dismissed. This risk is likely to be modest (as it is for R77H), supported by the identification of $\mathrm{F} 941 \mathrm{~V}$, which has the greatest impact on CR3 function, in unaffected family members through capillary sequencing.

Finally, both patients with ITGAM rare variants described here were included as part of a recent SLE GWAS (J. Bentham, D. L. Morris, et al., 2014, unpublished data) and their risk scores (allele count* $\ln (\mathrm{OR})$ ) fall within $1 \mathrm{SD}$ of the mean risk score for all cases (personal communication). If a relatively small number of rare variants were to contribute to large amount of the genetic variance of SLE, we might expect the two patients' risk scores, obtained from their genomic composition of associated common variants, to be relatively low. Together with the comparable functional impact of G1145S and R77H, our work suggests that some individual rare variants may contribute less to the genetic variance of complex diseases than is currently hypothesized, but it is possible that, on a population-wide scale, the presence of individual rare variants with weak effect size is a common phenomenon and, in total, accounts for much missing heritability, despite the current lack of appropriate statistical tools. As we have demonstrated, if they were located in known susceptibility loci and if the right functional assay were available, they might be relatively easy to find. The majority, however, are likely to remain undiscovered.

As well as demonstrating a general aspect of rare-variant discovery, our data also expand on our specific understanding of the role of ITGAM variants in SLE pathogenesis. First, we have resolved some uncertainty in the genetic 
literature concerning the possible additional functional effect of ITGAM SNP rs1143683 (A858V). SNP rs1143683 (A858V) and rs1143679 (R77H) are in relatively high LD $\left(r^{2}=0.55\right)$ in European populations, and rs1143683 has been suggested as a secondary independent association signal within ITGAM in a Thai population [22]. We wished to clarify this issue by including the A858V polymorphism in our in vitro model, along with another polymorphism, rs11861251 (M441T), which is not a part of the same disease-associated haplotype $\left(r^{2}=0.017\right)$, which we envisaged as a negative control. We found that neither the A858V nor the M441T mutations affect the CR3mediated phagocytosis of iC3b-coated targets.

A recent publication demonstrated a functional impact of ITGAM polymorphisms on ex vivo neutrophil functions, independent of $\mathrm{R} 77 \mathrm{H}$ [44]. There is perfect LD between rs1143683 (A858V) and rs1143678 (P1146S), and for this reason, the functional impact of these variants could be analyzed only on a haplotype basis. Even though they report a significant reduction in phagocytosis in individuals with A858V/P1146S variant neutrophils, they hypothesize that the affect is attributable to the less-conservative P1146S amino acid change. Our results support this hypothesis of the functional neutrality of A858V. Of particular interest, the patient who harbors the G1145S rare variant is also heterozygous for the P1146S polymorphism. Through using the IGV program to view aligned 454 sequencing reads, we can see that the G1145S rare variant and $\mathrm{P} 1146 \mathrm{~S}$ polymorphism are in phase on the chromosome. G1145S had the least severe impact on phagocytic function (26\% reduction); however, a functional alteration to two sequential amino acids could indeed have an impact greater than the additive effect of the G1145S and P1146S variants considered independently. This patient is also heterozygous for the associated $\mathrm{R} 77 \mathrm{H}$ polymorphism (of note, the patient with F941V is homozygous WT), so it is possible that the CR3 functions of this person are severely affected in vivo. The phenomenon of an additive functional effect of both rare and common variants within the same polypeptide molecule is of particular interest when considering the contribution of rare variants to complex disease susceptibility.

Our functional results also emphasize that impaired phagocytosis of iC3b opsonized particles, a consequence of defective "outside-in" signaling is one, but clearly not necessarily the only, functional mechanism underlying the association between specific ITGAM risk variants and SLE. It is notable that all three functional variants, $\mathrm{R} 77 \mathrm{H}, \mathrm{F} 941 \mathrm{~V}$, and G1145S, are located in different domains of the CD11b polypeptide (Figure 1) but have similar effects on signaling. None appears directly to influence known ligand-binding sites [45], but clearly specific amino acids throughout CD11b, from the N-terminal $\beta$-propeller (R77) domain through to the C-terminal cytoplasmic tail (G1145), are important in mediating CR3 outside-in signals to the cytoplasm. As seen with $\mathrm{R} 77 \mathrm{H}$, variants can affect function even if not in classically identified-ligand binding or intracellular signaling domains. This effect may be due to necessary interactions between CD11b and CD18 or due to the requirement for large conformational changes occurring during integrin activation/signaling [46].

The "hypothesis-free" GWAS method has proven successful in highlighting novel disease-associated polymorphisms for further functional analysis, but lacks statistical power to prove the association of most rare variants with disease. Recent approaches have identified rare variants in disease-associated loci very successfully, but estimating their contribution to disease risk has proven difficult. Conclusions are leading toward the notion that rare variants contribute little toward genetic variance [15]. We demonstrated that once the functional consequence of a common variant has been clearly elucidated, this knowledge can be reapplied to provide proof of a role for additional candidate rare variants at the same locus. In a relatively small resequencing project, we used this approach to identify two functional rare variants at the ITGAM locus. This study suggests that further screening of ITGAM for rare variants in a larger cohort of SLE cases promises to be worthwhile. If an association-based approach alone were applied in this study, there would be no evidence of any potential contribution of these rare variants to SLE pathogenesis. However, it is not possible to disregard these variants, given their genetic location, conservation, and deleterious effects on receptor function. If each of the 52 known SLEsusceptibility loci contains a similar high number of modest effect-size rare variants, then their contribution to the overall genetic component of disease susceptibility will be not inconsiderable. However, perhaps we currently lack the correct tools for estimating the contribution of rare variation to complex disease susceptibility.

\section{Conclusion}

We have identified two novel rare variants in the SLE susceptibility gene, ITGAM, and demonstrated their functional effects. However, because of their extremely low frequencies, they are not statistically associated with disease risk. This study questions the current notion of dismissing the contribution of very rare variants on purely statistical analyses alone. Our results add further evidence to the functional importance of ITGAM in SLE pathogenesis through impaired phagocytosis.

\section{Additional files}

Additional file 1: Table S1. Primer sequences used to generate amplicons covering coding regions of ITGAM for 454 library preparation. 
Additional file 2: Figure S1. Rare variant validation by capillary sequencing. Electropherogram images showing the Sanger Sequencing validation of the two rare variants.

Additional file 3: Table S2. Percentage phagocytosis of WT and variant CD11b-transfected COS-7 cells. Raw data comparing the percentage phagocytosis of WT and variant CD11b-transfected cells across five independent assays.

\section{Abbreviations}

CR3: Complement receptor 3; GWAS: genome-wide association study: LD: linkage disequilibrium; SLE: systemic lupus erythematosus.

\section{Competing interests}

The authors declare that they have no competing interests.

\section{Authors' contributions}

ALR: study design, data collection and analysis, and manuscript drafting. ERAT: study design, data collection and analysis, and manuscript drafting. SB: data collection and analysis. LG: data collection and analysis. OO: data collection and analysis. TJA: study conception and critical revision of the manuscript. TJV: interpretation of data and critical revision of the manuscript. BR: study conception and design, interpretation of data, and critical revision of the manuscript. All authors read and approved the final manuscript.

\section{Acknowledgements}

This work was supported by Arthritis Research UK (19317 to A.L.R, 18544 to B.R) and the Wellcome Trust (085260/Z/08/Z to E.R.A.T). We thank Tomas Axelson and Ann-Christine Syvänen, Uppsala University, and David L. Morris and James Bentham, King's College London, for the genotyping and analysis of the Illumina Custom 384 Chip, respectively. We acknowledge support from both the Imperial Biomedical Research Centre and National Institute for Health Research Biomedical Research Centre at Guy's and St Thomas' NHS Foundation Trust.

\section{Author details}

'Department of Medical and Molecular Genetics, and Inflammation, Infection and Immunity, King's College London, London SE1 9RT, UK. ${ }^{2}$ MRC Clinical Sciences Centre, Imperial College London, London, UK.

Received: 15 October 2013 Accepted: 7 May 2014

Published: 21 May 2014

\section{References}

1. Deapen D, Escalante A, Weinrib L, Horwitz D, Bachman B, Roy-Burman P, Walker AMT: A revised estimate of twin concordance in systemic lupus erythematosus. Arthritis Rheum 1992, 35:311-318.

2. Harley JB, Alarcón-Riquelme ME, Criswell L a, Jacob CO, Kimberly RP, Moser KL, Tsao BP, Vyse TJ, Langefeld CD, Nath SK, Guthridge JM, Cobb BL, Mirel DB, Marion MC, Williams AH, Divers J, Wang W, Frank SG, Namjou B, Gabriel SB, Lee AT, Gregersen PK, Behrens TW, Taylor KE, Fernando M, Zidovetzki R, Gaffney PM, Edberg JC, Rioux JD, Ojwang JO, et al: Genome-wide association scan in women with systemic lupus erythematosus identifies susceptibility variants in ITGAM, PXK, KIAA1542 and other loci. Nat Genet 2008, 40:204-210.

3. Moser KL, Kelly J, Lessard CJ, Harley JB: Recent insights into the genetic basis of systemic lupus erythematosus. Genes Immun 2009, 10:373-379.

4. Reich DE, Lander ES: On the allelic spectrum of human disease. Trends Genet 2001, 17:502-510.

5. Manolio TA, Collins FS, Cox NJ, Goldstein DB, Hindorff LA, Hunter DJ, McCarthy MI, Ramos EM, Cardon LR, Chakravarti A, Cho JH, Guttmacher AE, Kong A, Kruglyak L, Mardis E, Rotimi CN, Slatkin M, Valle D, Whittemore AS, Boehnke M, Clark AG, Eichler EE, Gibson G, Haines JL, Mackay TFC, McCarroll SA, Visscher PM: Finding the missing heritability of complex diseases. Nature 2009, 461:747-753.

6. Eichler EE, Flint J, Gibson G, Kong A, Leal SM, Moore JH, Nadeau JH: Missing heritability and strategies for finding the underlying causes of complex disease. Nat Rev Genet 2010, 11:446-450.

7. So H-C, Gui AHS, Cherny SS, Sham PC: Evaluating the heritability explained by known susceptibility variants: a survey of ten complex diseases. Genet Epidemiol 2011, 35:310-317.
8. Pritchard JK: Are rare variants responsible for susceptibility to complex diseases? Am J Hum Genet 2001, 69:124-137.

9. Pritchard JK, Cox NJ: The allelic architecture of human disease genes: common disease-common variant... or not? Hum Mol Genet 2002. 11:2417-2423

10. Heinzen EL, Depondt C, Cavalleri GL, Ruzzo EK, Walley NM, Need AC, Ge D, He M, Cirulli ET, Zhao Q, Cronin KD, Gumbs CE, Campbell CR, Hong LK, Maia JM, Shianna KV, McCormack M, Radtke R, O'Conner GD, Mikati M, Gallentine WB, Husain AM, Sinha SR, Chinthapalli K, Puranam RS, McNamara JO, Ottman R, Sisodiya SM, Delanty N, Goldstein DB: Exome sequencing followed by large-scale genotyping fails to identify single rare variants of large effect in idiopathic generalized epilepsy. Am J Hum Genet 2012, 91:293-302.

11. Keinan A, Clark AG: Recent explosive human population growth has resulted in an excess of rare genetic variants. Science 2012, 336:740-743.

12. Cooper GM, Shendure J: Needles in stacks of needles: finding disease-causal variants in a wealth of genomic data. Nat Rev Genet 2011, 12:628-640.

13. Torgerson DG, Capurso D, Mathias R, Graves PE, Hernandez RD, Beaty TH, Bleecker ER, Raby B, Meyers D, Barnes KC, Weiss ST, Martinez FD, Nicolae DL, Ober C: Resequencing candidate genes implicates rare variants in asthma susceptibility. Am J Hum Genet 2012, 90:273-281.

14. Rivas M, Beaudoin M, Gardet A, Stevens C, Sharma Y, Zhang CK, Boucher $G$ Ripke S, Ellinghaus D, Burtt N, Fennell T, Kirby A, Latiano A, Goyette P, Green T, Halfvarson J, Haritunians T, Korn JM, Kuruvilla F, Lagacé C, Neale B, Lo KS, Schumm P, Törkvist L, Dubinsky MC, Brant SR, Silverberg MS, Duerr RH, Altshuler D, Gabriel S, et al: Deep resequencing of GWAS loci identifies independent rare variants associated with inflammatory bowel disease. Nat Genet 2011, 43:1066-1073.

15. Hunt K, Mistry V, Bockett N, Ahmad T, Ban M, Barker JN, Barrett JC, Blackburn H, Brand O, Burren O, Capon F, Compston A, Gough SCL, Jostins L, Kong Y, Lee JC, Lek M, MacArthur DG, Mansfield JC, Mathew CG, Mein C, Mirza M, Nutland S, Onengut-Gumuscu S, Papouli E, Parkes M, Rich SS, Sawcer S, Satsangi J, Simmonds MJ, et al: Negligible impact of rare autoimmune-locus coding-region variants on missing heritability. Nature 2013, 498:232-235.

16. Han S, Kim-Howard X, Deshmukh H, Kamatani Y, Viswanathan P, Guthridge JM, Thomas K, Kaufman KM, Ojwang J, Rojas-Villarraga A, Baca V, Orozco L, Rhodes B, Choi C-B, Gregersen PK, Merrill JT, James J, Gaffney PM, Moser KL, Jacob CO, Kimberly RP, Harley JB, Bae S-C, Anaya J-M, Alarcón-Riquelme ME, Matsuda K, Vyse TJ, Nath SK: Evaluation of imputation-based association in and around the integrin-alpha-M (ITGAM) gene and replication of robust association between a non-synonymous functional variant within ITGAM and systemic lupus erythematosus (SLE). Hum Mol Genet 2009, 18:1171-1180.

17. Ross GD, Vĕtvicka V: CR3 (CD11b, CD18): a phagocyte and NK cell membrane receptor with multiple ligand specificities and functions. Clin Exp Immunol 1993, 92:181-184.

18. Nath SK, Han S, Kim-Howard X, Kelly J, Viswanathan P, Gilkeson GS, Chen W, Zhu C, McEver RP, Kimberly RP, Alarcón-Riquelme ME, Vyse TJ, Li Q-Z, Wakeland EK, Merrill JT, James J, Kaufman KM, Guthridge JM, Harley JB: A nonsynonymous functional variant in integrin-alpha(M) (encoded by ITGAM) is associated with systemic lupus erythematosus. Nat Genet 2008, 40:152-154.

19. Rhodes B, Fürnrohr BG, Roberts AL, Tzircotis G, Schett G, Spector TD, Vyse TJ: The rs 1143679 (R77H) lupus associated variant of ITGAM (CD11b) impairs complement receptor 3 mediated functions in human monocytes. Ann Rheum Dis 2012, 71:2028-2034.

20. Fossati-Jimack L, Ling GS, Cortini A, Szajna M, Malik TH, McDonald JU, Pickering MC, Cook HT, Taylor PR, Botto M: Phagocytosis is the main CR3-mediated function affected by the lupus-associated variant of CD11b in human myeloid cells. PLOS One 2013, 8:e57082.

21. MacPherson M, Lek HS, Prescott A, Fagerholm SC: A systemic lupus erythematosus-associated R77H substitution in the CD11b chain of the Mac-1 integrin compromises leukocyte adhesion and phagocytosis. J Biol Chem 2011, 286:17303-17310.

22. Yang W, Zhao M, Hirankarn N, Lau CS, Mok CC, Chan TM, Wong RWS, Lee KW, Mok MY, Wong SN, Avihingsanon Y, Lin IO, Lee TL, Ho MHK, Lee PPW, Wong WHS, Sham PC, Lau YL: ITGAM is associated with disease susceptibility and renal nephritis of systemic lupus erythematosus in Hong Kong Chinese and Thai. Hum Mol Genet 2009, 18:2063-2070.

23. Ke X: Presence of multiple independent effects in risk loci of common complex human diseases. Am J Hum Genet 2012, 91:185-192. 
24. Meyer M, Stenzel U, Hofreiter M: Parallel tagged sequencing on the 454 platform. Nat Protoc 2008, 3:267-278.

25. Quinlan AR, Stewart DA, Marth T: Pyrobayes: an improved base caller for SNP discovery in pyrosequences. Nat Methods 2008, 5:454-456.

26. Wang L, Gordon R, Huynh L, Su X, Park Min K-H, Han J, Arthur JS, Kalliolias $\mathrm{GD}$, Ivashkiv LB: Indirect inhibition of Toll-like receptor and type I interferon responses by ITAM-coupled receptors and integrins. Immunity 2010, 32:518-530.

27. McKenna A, Hanna M, Banks E, Sivachenko A, Cibulskis K, Kernytsky A, Garimella K, Altshuler D, Gabriel S, Daly M, DePristo M: The Genome Analysis Toolkit: a MapReduce framework for analyzing next-generation DNA sequencing data. Genome Res 2010, 20:1297-1303.

28. Caron E: Identification of Two Distinct Mechanisms of Phagocytosis Controlled by Different Rho GTPases. Science (80-) 1998, 282:1717-1721.

29. Wiedemann A, Patel JC, Lim J, Tsun A, van Kooyk Y, Caron E: Two distinct cytoplasmic regions of the beta2 integrin chain regulate RhoA function during phagocytosis. J Cell Biol 2006, 172:1069-1079.

30. Adzhubei I, Schmidt S, Peshkin L, Ramensky VE, Gerasimova A, Bork P, Kondrashov AS, Sunyaev SR: A method and server for predicting damaging missense mutations. Nat Methods 2010, 7:248-249.

31. Kumar P, Henikoff S, Ng PC: Predicting the effects of coding non-synonymous variants on protein function using the SIFT algorithm. Nat Protoc 2009, 4:1073-1081.

32. 1000 Genomes Project. [http://www.1000genomes.org/]

33. NHLBI Exome Sequencing Project. [http://evs.gs.washington.edu/EVS/]

34. Corpet F, Cellulaire LDG, Toulouse I, Tolosan C: Multiple sequence alignment with hierarchical clustering. Nucleic Acids Res 1988 16:10881-10890.

35. Multiple Sequence Alignment. [http://multalin.toulouse.inra.fr/multalin/]

36. UCSC Genome Browser. [http://genome.ucsc.edu/]

37. SNP Annotation and Proxy Search. [http://www.broadinstitute.org/mpg/ snap/ldsearchpw.php]

38. Jordan CT, Cao L, Roberson EDO, Duan S, Helms C, Nair RP, Duffin KC, Stuart PE, Goldgar D, Hayashi G, Olfson EH, Feng B-J, Pullinger CR, Kane JP, Wise C, Goldbach-Mansky R, Lowes M, Peddle L, Chandran V, Liao W, Rahman P, Krueger GG, Gladman D, Elder JT, Menter A, Bowcock AM: Rare and common variants in CARD14, encoding an epidermal regulator of NF-kappaB, in psoriasis. Am J Hum Genet 2012, 90:796-808.

39. Need AC, McEvoy JP, Gennarelli M, Heinzen EL, Ge D, Maia JM, Shianna KV, He M, Cirulli ET, Gumbs CE, Zhao Q, Campbell CR, Hong L, Rosenquist P, Putkonen A, Hallikainen T, Repo-Tiihonen E, Tiihonen J, Levy DL, Meltzer HY, Goldstein DB: Exome sequencing followed by large-scale genotyping suggests a limited role for moderately rare risk factors of strong effect in schizophrenia. Am J Hum Genet 2012, 91:303-312.

40. Witte T, Dumoulin FL, Gessner JE, Schubert J, Götze O, Neumann C, Todd RF, Deicher H, Schmidt RE: Defect of a complement receptor 3 epitope in a patient with systemic lupus erythematosus. J Clin Invest 1993, 92:1181-1187.

41. Bodmer W, Bonilla C: Common and rare variants in multifactorial susceptibility to common diseases. Nat Genet 2008, 40:695-701.

42. Herrmann M, Voll RE, Zoller OM, Hagenhofer M, Ponner BB KJ: Impaired phagocytosis of apoptotic cell material by monocyte-derived macrophages from patients with systemic lupus erythematosus. Arthritis Rheum 1998, 41:1241-1250.

43. Han C, Jin J, Xu S, Liu H, Li N, Cao X: Integrin CD11b negatively regulates TLR-triggered inflammatory responses by activating Syk and promoting degradation of MyD88 and TRIF via Cbl-b. Nat Immunol 2010, 11:734-742.
44. Zhou Y, Wu J, Kucik DF, White NB, Redden DT, Szalai AJ, Bullard DC, Edberg JC: Multiple lupus-associated ITGAM variants alter Mac-1 functions on neutrophils. Arthritis Rheum 2013, 65:2907-2916.

45. Diamond MS, Garcia-Aguilar J, Bickford JK, Corbi aL, Springer T: The domain is a major recognition site on the leukocyte integrin Mac-1 (CD11b/CD18) for four distinct adhesion ligands. J Cell Bio/ 1993, 120:1031-1043.

46. Lefort $C T$, Hyun Y-M, Schultz JB, Law F-Y, Waugh RE, Knauf P, Kim M: Outside-in signal transmission by conformational changes in integrin Mac-1. J Immunol 2009, 183:6460-6468.

doi:10.1186/ar4566

Cite this article as: Roberts et al:: Resequencing the susceptibility gene, ITGAM, identifies two functionally deleterious rare variants in systemic lupus erythematosus cases. Arthritis Research \& Therapy 2014 16:R114.

\section{Submit your next manuscript to BioMed Central and take full advantage of:}

- Convenient online submission

- Thorough peer review

- No space constraints or color figure charges

- Immediate publication on acceptance

- Inclusion in PubMed, CAS, Scopus and Google Scholar

- Research which is freely available for redistribution

Submit your manuscript at www.biomedcentral.com/submit
C Biomed Central 